\title{
Bilateral Clinical Pathology of the Temporal Bone
}

\author{
Miriam I. Saadia-Redleaf*
}

Department of Otolaryngology-Head and Neck Surgery, The University of Illinois at Chicago, Chicago, IL 60612, USA

\begin{abstract}
Objectives/Hypothesis: The aim of this study is to indicate anatomic and immunologic peculiarities of the temporal bone which leave it vulnerable to traumatic, infectious and neoplastic insult.

Study Design: Seven patients' cases are described in which the clinical pathology of the temporal bone was bilateral. The bilaterality of these cases serves to illustrate the anatomic points which are the focus of this study.

Methods: This is a case series.

Results: The seven paradigmatic cases are described and the relevant anatomy is explored. These are bilateral tuberculosis of the middle ear and mastoid, bilateral histiocytosis X, bilateral external canal amyloidosis, bilateral middle ear polyposis in Samter's triad, bilateral temporal bone fractures, bilateral middle cranial fossa encephaloceles, and bilateral congenital enlarged superior vestibular nerve canals. The thinness of the skull base, the many fluid-filled and tissue-filled canals which enter the temporal bone and the thinness of the tissues investing all the surfaces contribute to the temporal bone's vulnerability to trauma, infection and neoplasm.
\end{abstract}

Conclusion: Cases of bilateral temporal bone pathology demonstrate the temporal bone's structural and immunologic weaknesses.

Keywords: Temporal bone anatomy, temporal bone pathology, temporal bone trauma, temporal bone neoplasm, temporal bone inflammatory process.

\section{INTRODUCTION}

This thesis maintains that specific unique anatomic features and immunologic vulnerabilities make the temporal bones peculiarly susceptible to disease. As a result, both temporal bones and their contents frequently harbor the same disease process simultaneously. Otitis media and mastoiditis are common. Neurofibromatosis II and post-meningitic ossification of the cochlea (labyrinthitis ossificans) $[1,2]$ are well recognized. A review of patients over the last decade finds several other disease processes which arose in both ears. These were bilateral tuberculosis of the mastoids and middle ears [3], histiocytosis $\mathrm{X}$ of the mastoids and middle ear spaces, amyloidosis of the external auditory canals in multiple myeloma [4], bilateral middle ear polyposis in a patient with samter's triad, hairline transverse temporal bone fractures [5], bilateral middle cranial fossa encephaloceles presenting as conductive hearing loss, and bilateral malformations of the superior vestibular nerve canals in a patient with sensorineural hearing loss [6]. A recent series of malignant melanoma metastatic to the internal auditory canals reports that the metastases are bilateral in $4 / 7$ patients [7]. These unexpected bilateral manifestations of disease demonstrate the point of this thesis - that the temporal bone and its tissues are particularly vulnerable to disease. The anatomic and immunologic patterns of that vulnerability are outlined.

*Address correspondence to this author at the Department of Otolaryngology-Head and Neck Surgery, The University of Illinois at Chicago, 1855 W. Taylor Street, Chicago, IL 60612, USA; Tel: 312-9966583; Fax: 312-996-9910; E-mail: mredleaf@uic.edu

\section{SEVEN CASES OF BILATERAL TEMPORAL BONE PATHOLOGY}

Case \#1 - A 3 year old girl presented after several months of bilateral ear drainage, recalcitrant to local medical treatment [3]. Her mother had recently noticed that the child's smile was asymmetric and that her gait was unsteady. On presentation profuse white granulation tissue obscured both tympanic membranes. The post auricular mastoid skin was unremarkable. She had left sided facial palsy and an ataxic gait. Ear cultures grew out staphylococcus species and aspergillus niger. Computed tomography (CT) of the temporal bones showed bilateral soft tissue densities in the mastoids and middle ears This process had eroded the right tegmen tympani, ossicles, and scutum. On the left the destruction was less pronounced. The patient underwent right radical mastoidectomy and left modified radical mastoidectomy. Profuse while debris filled the entire external auditory canals and middle ears, with erosion of the bone over the cerebellar dura, carotid artery, and the sigmoid sinus. Gram stain and culture of the debris were negative, but an acid-fast stain was positive (Fig. 1).

Subsequent brain magnetic resonance imaging (Fig. 2) found several intraparenchymal ring-enhancing lesions. The patient was treated presumptively with isoniazid, rifampin, pyrazinamide, and streptomycin. Culture grew out mycobacterium tuberculosis 4 weeks later. Ear drainage stopped after a few weeks of anti-tubercular medications. The patient continued to make steady neurologic progress over the ensuing months. 


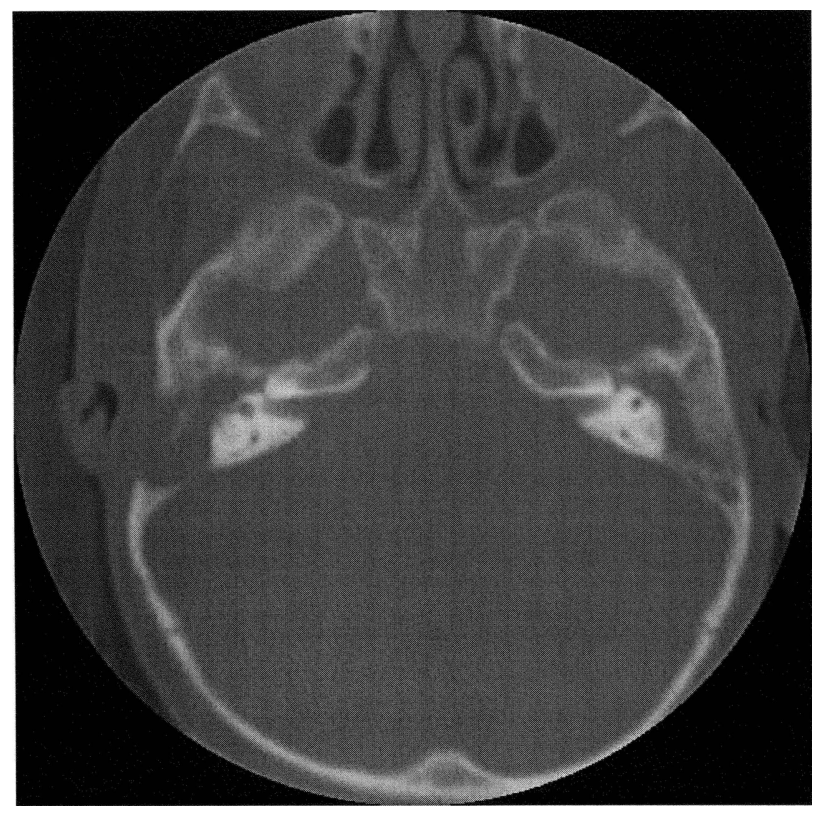

Fig. (1). Pre-operative axial computed tomography of the temporal bones of case \#1, a 3 year old girl with tuberculous mastoiditis and intracerebral foci. On mastoidectomy, the tuberculous debris filled bilateral middle ear spaces and mastoids and eroded the boney covers of the carotid arteries, sigmoid sinuses, and cerebellar plates.

Case \#2 - This pediatric patient was diagnosed with Langerhans cell histiocystosis at 10 months, after she developed weight loss and rash. Vincristine and etoposide treatment failed to prevent liver involvement, and 6mercaptopurine, methotrexate, and prednisone were added. From 12 months of age the patient's ears were draining bilaterally. Many culture-directed antibiotic regimen and sets of tube did not eliminate her drainage. CT of the temporal bones (Fig. 3) consistently demonstrated soft tissue densities in the middle ears and mastoid despite all interventions, and despite the waxing and waning of her histiocytosis loci. Clinically the canals began to fill with soft tissue, even as her liver focus was disappearing. At 3 years old she underwent bilateral mastoidectomies. Pathologic evaluation found histiocytosis in all samples from the mastoids, middle ear and ear canals. After her surgeries, soft tissue returned in both canals within weeks. At the age of 5 her visceral lesions began to regress. At the age of 6 , her temporal bone sites began to regress, evidently part of this disease's natural history, opening her ear canals.

Case \#3 - A 47 year old woman came in twice for removal of cerumen impactions [4]. Before her third visit for the same problem she developed systemic symptoms which led to the diagnosis of multiple myeloma with monoclonal lambda light-chains in the serum and urine. Her ear canals particularly the meatuses - showed friable, heaped up canal skin (Fig. 4). Bilateral biopsies bilaterally showed dermal amyloidosis. She underwent treatment for her systemic disease, while her ear canals were treated symptomatically. As her multiple myeloma regressed, her ear canals opened more widely.

Case \#4 - A 52 year-old female, with history of samter's triad, treated twice previously with functional endoscopic sinus surgery for recurrent nasal polyposis, returned for evaluation of ongoing right otalgia, otorrhea, and hearing loss. A large purulent polyp completely filled the right ear canal, while the left tympanic membrane showed a posterior superior polyp. A biopsy of the right polyp was consistent with pyogenic granuloma. Computed tomography showed bilateral soft tissue disease in the mastoids as well as the middle ear space, without boney erosion, and extending into the external auditory canal on the right (Fig. 5).

The patient then underwent a right tympanomastoidectomy with numerous polyps identified during dissection within the sinodural angle, epitympanum, and the remaining middle ear space. No evidence of keratin debris was found during the dissection. The histopathology was reported as mucous containing, inflammatory otic polyps with eosinophila and Charcot-Lyden crystals. Findings consistent with cholesteatoma were not observed. Postoperatively, the patient's middle ear mucosal hypertrophy recurred, and was treated successfully with intratympanic steroids.

Case \#5 - A 28 year old body builder worked the afternoon shift, drank a high energy drink containing

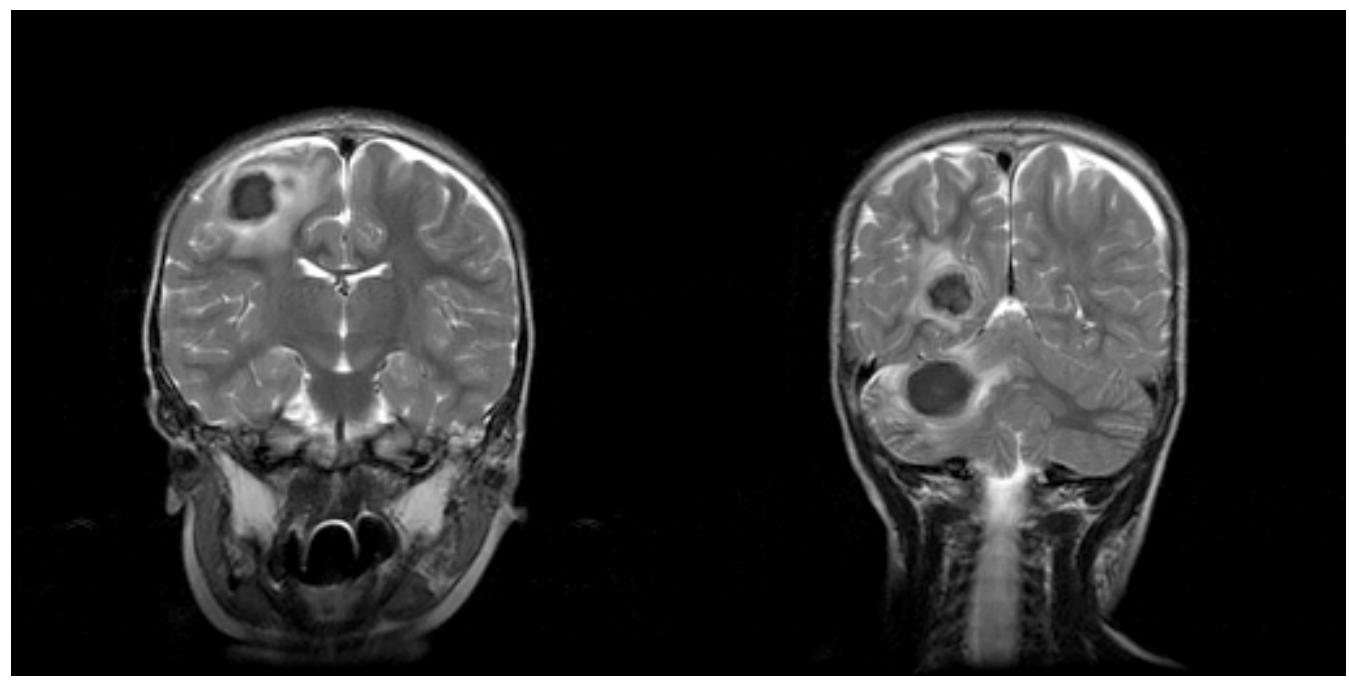

Fig. (2). T -2 weighted magnetic resonance imaging of the brain of case \#1, showing some of the intraparenchymal TB abscesses. 


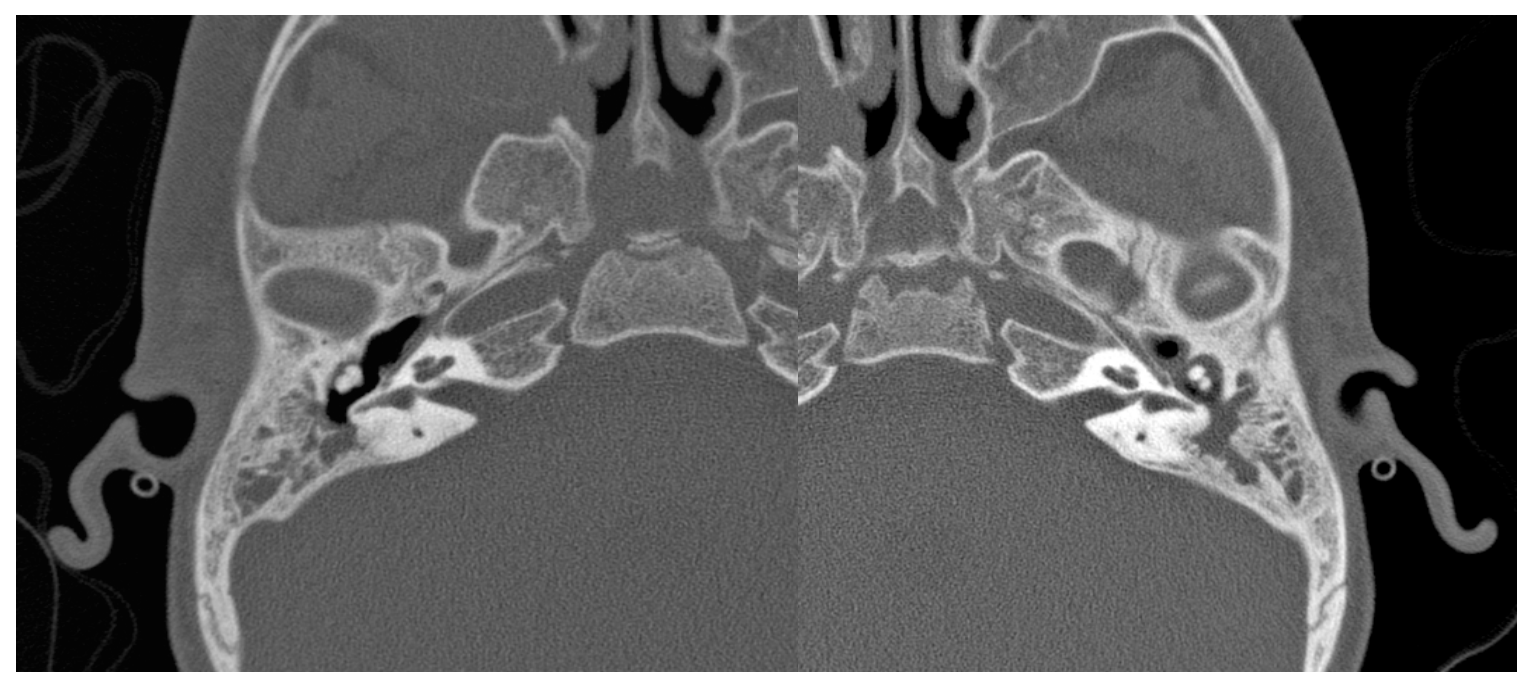

Fig. (3). Axial computed tomography of the temporal bones of case \#2, a 5 year old girl with systemic histiocytosis X, affecting the liver, and scalp. On mastoidectomy, histiocytosis $\mathrm{X}$ was present in bilateral mastoids and middle ears.

ephedrine, synephrine, and caffeine, and went to an all night gym [5]. At 2:30 am he was doing hyperextension exercises of the back, when he seized, hit his head, and upon regaining consciousness, immediately stated he could not hear. Physical exam found bilateral hemotympanum. Audiogram showed bilateral profound sensorineural hearing loss. Temporal bone CT showed bilateral hairline fractures through the cochleae (Fig. 6). The patient subsequently underwent successful cochlear implantation.

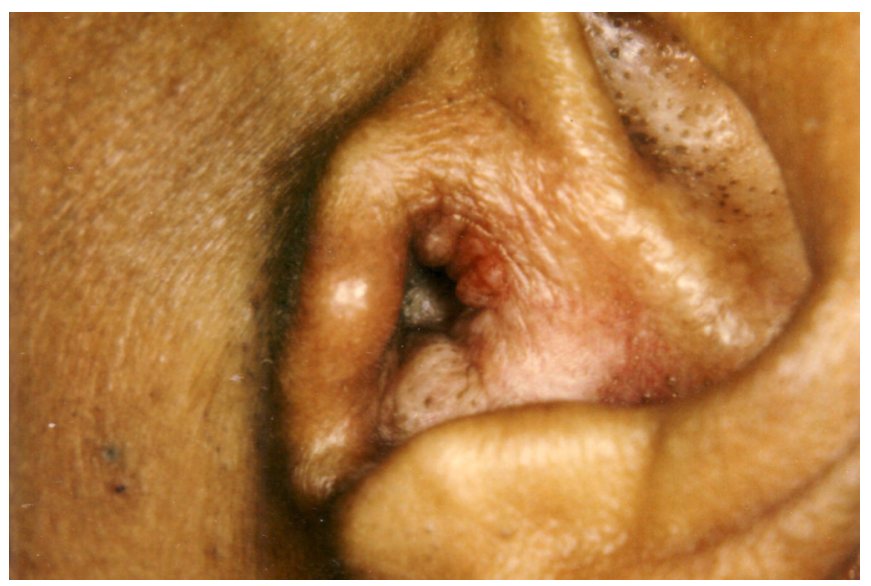

Fig. (4). Amyloidosis of bilateral external auditory canals in case \#3, a patient with multiple myeloma. Close up view of the left external canal meatus.

Case \#6 - A 47 year old obese diabetic woman presented with several years of hearing loss. On physical examination her tympanic membranes were opaque and immobile, while audiogram showed a bilateral mixed hearing loss with flat tympanograms. CT of the temporal bones showed bilateral soft tissue densities of the middle ears and mastoids. Bilateral mastoiditis was suspected, however upon raising tympanomeatal flaps bilaterally, well encapsulated soft tissue masses were found. When these were biopsied well away from the location of the facial nerve, pathology revealed CNS neuroglial tissue, confirmed by neuronspecific enolase staining. Since encephalocele was now suspected, an MRI was obtained which confirmed transgression by neuronal tissue of the middle cranial fossa floor (Fig. 7). The patient then underwent right tympanomastoidectomy with repair of the middle cranial fossa floor but was lost to follow up before her left ear could be addressed.

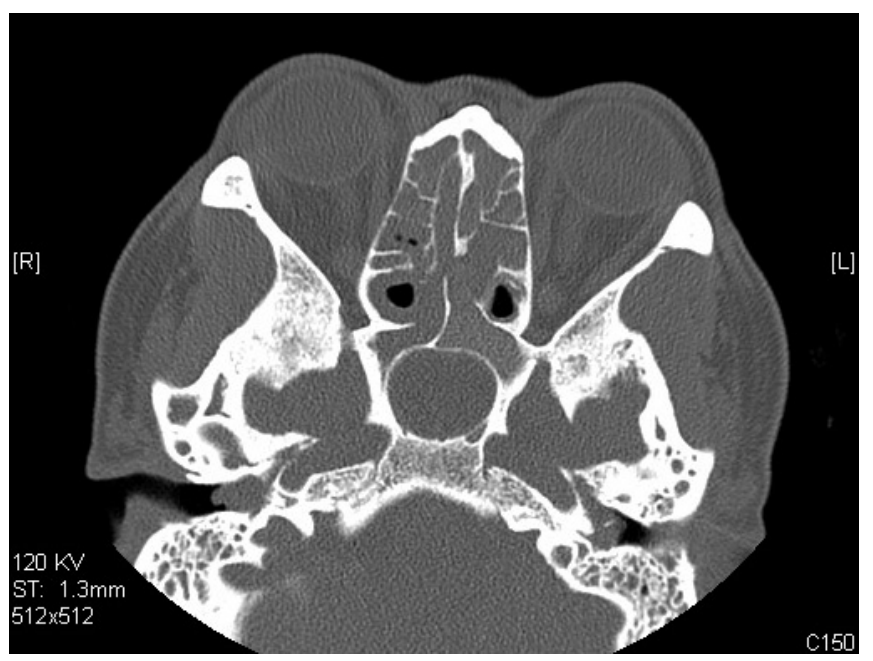

Fig. (5). Computed tomography of case \#4- samter's triad polyposis - showing soft tissue filling both middle ear spaces and protruding into the right external auditory canal.

Case \#7 -A 10 month old boy failed his newborn hearing screening test [6]. Physical exam was unremarkable. Auditory brainstem response showed no waves detected below $70 \mathrm{~dB}$ on the left and none detected below $80 \mathrm{~dB}$ on the right. Temporal bone CT (Fig. 8) showed bilaterally enlarged superior vestibular nerve canals and bilateral distortion of the vestibules at the nerve entry site. The superior vestibular nerve canal length and width at the most medial portion (ie, at their exit from the internal auditory canal) were measured from the temporal bone CT using the Centricity DICOM viewer software. The right canal's length was $2.28 \mathrm{~mm}$, and its width was $2.24 \mathrm{~mm}$. The left canal's length was $2.74 \mathrm{~mm}$ and its width was $1.82 \mathrm{~mm}$. (Normal lengths and widths of the superior vestibular nerve canal are approximately $1.9-2.3 \mathrm{~mm}$ and $1.04 \mathrm{~mm}$ respectively $[8,9])$. 


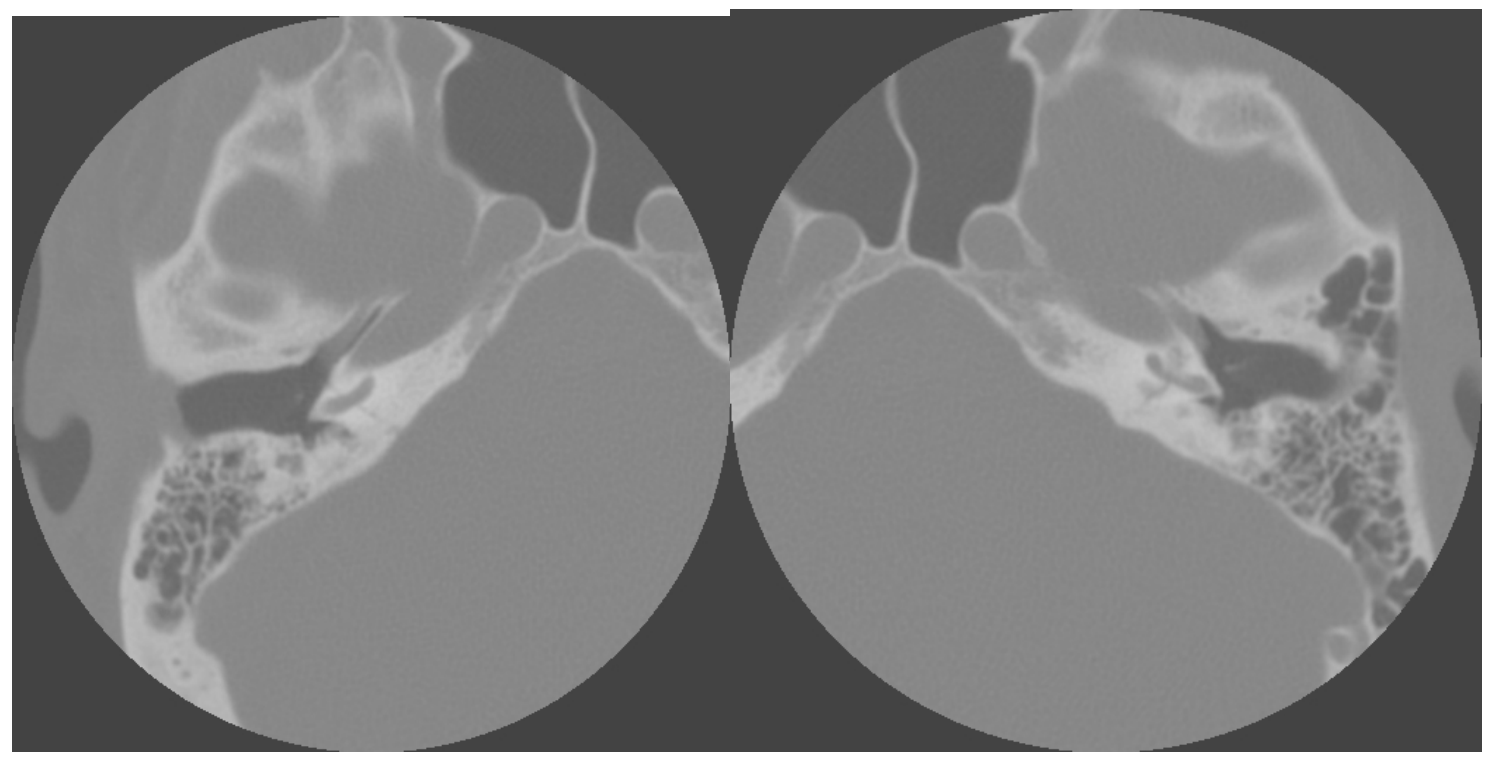

Fig. (6). Axial computed tomography of bilateral transcochlear hairline fractures in case \#5, after a single blow to the head.

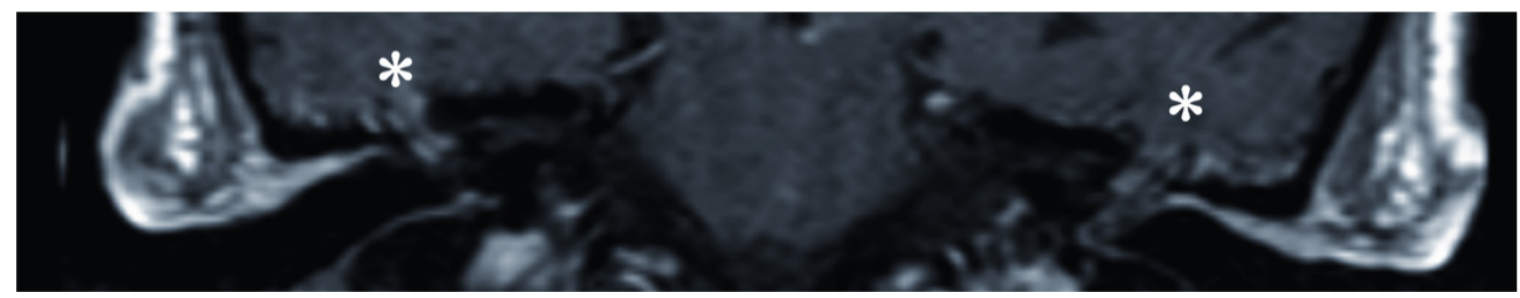

Fig. (7). T1-weighted coronal MRI with gadolinium of the temporal bones and skull base of case \#6. Aterisk (*) indicates the areas of bilateral encephaloceles herniating through the middle cranial fossa floor.

Remaining structures including the facial nerve and internal auditory canals were normal. The patient was referred for amplification.

\section{DISCUSSION}

A variety of disease processes affect both ears simultaneously. Common bilateral disorders are otitis media, mastoiditis and presbycusis. Less common are neurofibromatosis II, and labyrinthitis ossificans as a sequela of meningitis. Case \#1 above reports bilateral simultaneous tubercular mastoiditis with loci of brain abscesses. Case \#2, of histocytosis $\mathrm{X}$, shows the bilaterality of the disease, as well at its complete non-response to the same chemotherapeutic agents that were shrinking the patient's visceral lesions. In case $\# 3$, of multiple myeloma, the amyloid deposition in the external auditory canals was the only cutaneous finding of systemic disease. Case \#4, of samter's triad of the middle ear with polyposis, shows the middle ear's close relationship to the nasal pathology via the eustachian tube's anatomic and mucosal connection. Case \#5's hairline transverse temporal bone fractures clearly demonstrate the structural weakness of the adult temporal bone, as does case \#6's bilateral encephaloceles. Case \#7 reports a congenital malformation of the superior vestibular nerve canals associated with bilateral sensorineural hearing loss, and serves as a reminder that developmental abnormalities, too, are usually bilateral. The temporal bone appears to be particularly vulnerable to infectious,

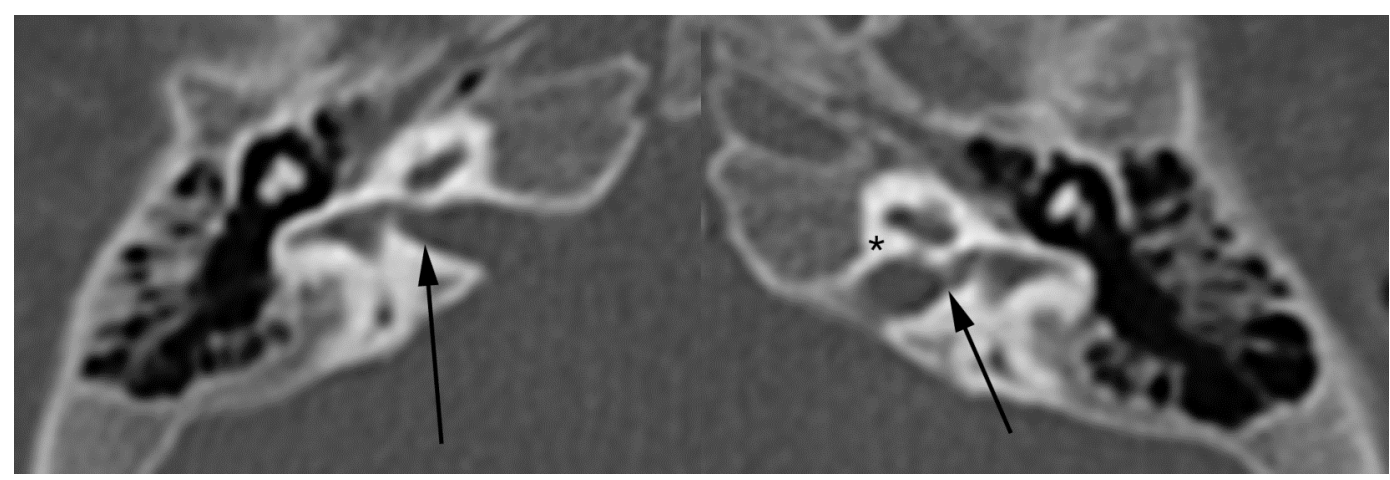

Fig. (8). Axial computed tomography of the temporal bones of case \#7 showing bilateral widening of the canals for the superior vestibular nerves (solid black arrows). Asterisk $\left.*^{*}\right)$ is directly adjacent to the left auditory nerve canal. 
neoplastic, and traumatic insults, hence the bilaterality of disease in these cases.

The unique anatomy of the temporal bone begins to explain some of this susceptibility to insult. This labyrinth is suspended in the honey combed trabeculae of the temporal bone, with five long canals radiating from it - the internal and external auditory canals, the cochlear aqueduct, and vestibular aqueduct, and the Eustachian tube-and-middle ear (Fig. 9). These canals are like spokes of a bicycle wheel, radiating out from the hub of the otic capsule and middle ear space. However, rather than provide structural support for the functional hub of audiologic activity where they converge, they weaken any support.

Blood supply along all these hollow surfaces is sparse: four of these canals are invested with either a thin soft tissue lining, or no lining at all. The eustachian tube-middle ear canal has a thin respiratory mucosal lining in its middle ear and otic portions, and a thick mucosal-musculocartilagenous investment in its nasopharyngeal portion. At any rate, no other boney canals in the skull base are as wide or as long as those penetrating the temporal bone. A clear example of the structural defensive defect of the canals can be seen in meningitis; the cochlear aqueduct itself virtually seems to usher the bacterial elements directly into the scala tympani of the cochlea where the end response is an obliterative ossification $[1,2]$.

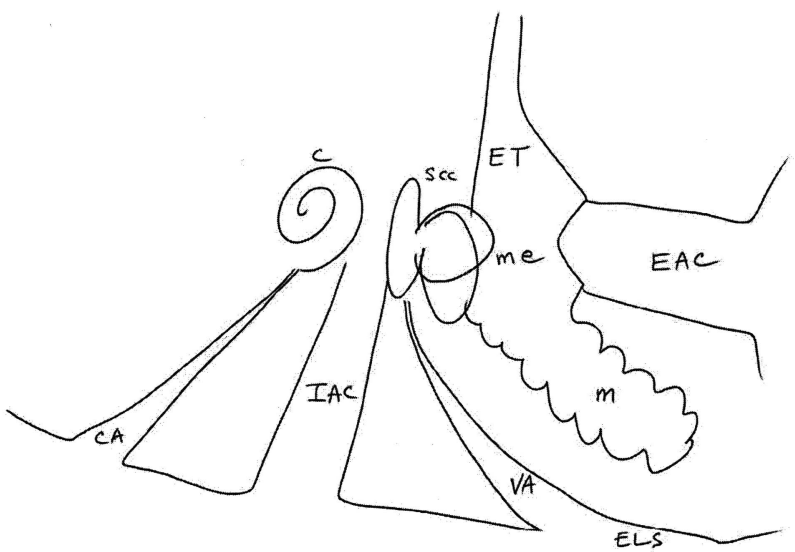

Fig. (9). Schematic of the 5 boney canals radiating out from the vestibulo-otic labyrinth. The internal auditory canal (IAC) is invested with a thin dural lining. The external auditory canal (EAC) is invested with a typically thin lining of skin. Vestibular aqueduct (VA) lining is variable. The endolymphatic sac (ELS) is more invested with vascularized tissue. The cochlear aqueduct (CA) is lined with periosteum. The eustachian tube (ET) is musculocartilagenous in its nasopharyngeal portion, and the middle ear (me) and mastoid $(\mathrm{m})$ have a thin lining of respiratory mucosa.

Another anatomic perspective shows the many additional structures perforating through the temporal bone, running adjacent to the otic capsule. The five long canals listed above are each filled with CSF or CSF ultrafiltrate or air. In addition to these hollow canals, there are canals which are filled with soft tissue or flowing blood. These are the canals for the exits of the auditory, vestibular and facial nerves from the internal canal; for the facial nerve's path; for the tensor tympani muscle; for the carotid artery; and for the jugular bulb and its exit (Fig. 10). These many perforations render the temporal bone much more susceptible to traumatic injury. The otic capsule itself is formed from endochondrial ossification and is neither robust against blows, nor able to reossify. Further, the middle cranial fossa contents are separated from the middle ear space and mastoid by a layer of bone which is usually thin at birth and often becomes dehiscent over the years. The patient reported as case \#5 shows how a single blow to the back of the head can fracture through both cochleae; and case \#5 redemonstrates that even a thin fracture through the otic capsule is functionally devastating. Case \#6 of bilateral encephaloceles demonstrates that the floor of the middle cranial fossa is thin and can give way to the intracranial contents' herniation into the middle ear and mastoid.

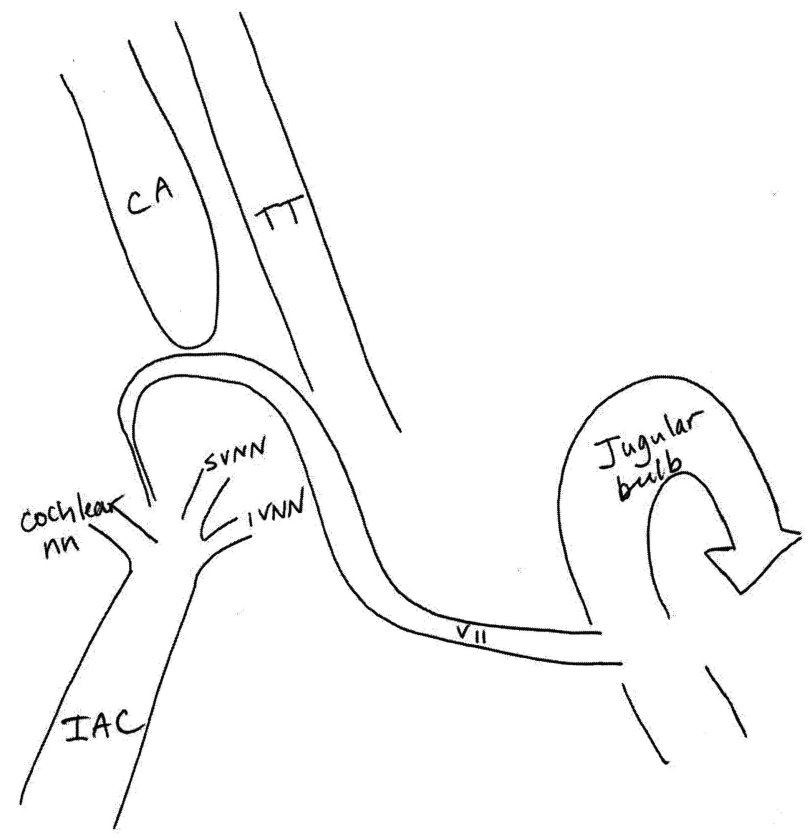

Fig. (10). Schematic of the local canals in the temporal bone. Crevices and canals which further interrupt the bone's continuity are the exits of the vestibular (SVNN and IVNN), cochlear and facial nerves; facial nerve route (VII); tensor tympani canal (TT); jugular bulb and carotid artery (CA) canals.

Case \#1 of TB of the temporal bone demonstrates that infectious processes travel freely in the temporal bone's canals and aqueducts and come to rest in the canals or in the adjacent cavities. Bacterial agents of meningitis appear to flourish preferentially in these sites, even though they are present in other areas of the brain and skull base. The mycobacteria of tuberculosis clearly are present in the entire body. Indeed, they apparently traversed case \#1's brain's parenchyma. Yet the tuberculosis settled in the mastoids and middle ear spaces bilaterally and symmetrically.

Case \# 4 had samter's triad and bilateral otic polyps with eosinophils and Charcot-Lyden crystals. The same kind of polyp was present in the middle ears as is found in the paranasal sinuses of samter's triad. Were the otic polyps present on the basis of direct extension from the nasopharynx, or were they the same process in the respiratory mucosa of the middle ear as in the sinus? Either way the case demonstrates the close functional or anatomic relationship between the middle ear and the nasopharynx. 
And it points out the susceptibility of the middle ear space to this inflammatory process.

The mode of dissemination of Histocytosis $\mathrm{X}$ is unknown. However, the ear canals and mastoids are common sites of involvement [10]. In case \#2, the disease settled and progressed in both mastoids and middle ear spaces. Further, in this case, the histiocytosis lesions in the temporal bone actually progressed during chemotherapy as the other visceral lesions were diminishing. So blood borne chemotherapeutic agents were ineffective in the ear, even while they were reducing the liver's involvement. Unfortunately for this patient, surgical removal of the disease also did not eradicate it, since the histiocytosis masses returned to the ear canals within weeks of surgery. Apparently the temporal bones have reduced resistance to pathogens or neoplasms that other areas of the body and intracranial vault are able to resist. Perhaps the meager blood supply as seen in the thinness of the dural and mucosal linings of the canals and aircell spaces is responsible for the temporal bone's poor resistance.

The temporal bone shows a similar susceptibility to metastasis from other neoplasms. Blood borne metastases from non-cranial portions of the body clearly are present in the systemic blood supply, traveling in the brain, meninges, and even the rest of the body. Yet metastases from below often come to rest and expand, relatively unopposed, in the internal auditory canals. Indeed, a recent report [7] of malignant melanoma metastases to the internal auditory canals supports this analysis: the authors of this series report that 4 of 7 patients with melanoma metastatic to the internal auditory canals had bilateral metastases. Interestingly, none of these 4 patients showed metastases elsewhere in the body or the central nervous system. Unfortunately, only 2 patients had CSF analysis. However, both of these 2 showed melanotic cells in the CSF. Therefore, in at least 2 of these cases, and perhaps all of them, the melanotic cells were present in the CSF and presumably also the blood, but only survived and grew in the temporal bones. Others also report cases of bilateral IAC metastases [11, 12].

Neurofibromatosis II may also be an example of the immune system's failure to suppress a neoplastic process. Neurofibromatosis II typically manifests many benign intracranial tumors in addition to the bilateral vestibular schwannomas. The precise locations of the other neoplasms vary from patient to patient while the intracanilicular tumors always arise. In other words, intracranial neoplasms spontaneously arise at many variable sites, and presumably some of these tumors are suppressed. But the internal auditory canals seem particularly unable to suppress them, just as the internal canals are unable to suppress the melanotic metastases. Hence the bilateral schwannomas become a hallmark of the disease.

The amyloid deposits seen in case \#3 are the deposition of protein components which had been overproduced in the condition of multiple myeloma. These can be deposited anywhere in the skin or viscera, but tend to congregate in the liver, spleen, kidney and heart. Clearly there must be some feature of the external auditory canal which favors protein deposition - the blood supply, the temperature, the thinness of the canal skin.
Many of these disease processes are also seen unilaterally in the temporal bone, of course. The bilateral manifestations only underscore that the temporal bone has unique weaknesses in its defenses. In addition, the same disease processes - TB, bacterial infections, histiocytosis, amyloidosis, and metastases are also seen in other body parts, but not symmetrically. Therefore disease manifests itself bilaterally in the temporal bone but not bilaterally in the rest of the body, once again suggesting that there is some weakness in the temporal bone. At the very least, the cases described here indicate that any condition which affects one temporal bone could likely affect the other one, and the clinician should look for bilateral involvement.

Although the anatomic and immune related factors appear to contribute to the vulnerability of the temporal bone to insult, there can be no generalizations about the best treatments for these. Congenital malformations of the temporal bone and otic capsule fractures which result in sensorineural hearing loss can only be treated with amplification or implantation (cases $\# 5$ and 7). Encephalocele with ossicular dysfunction is surgically addressed (case \#6). Histiocytosis and samter's polyposis do not respond to surgery, although the one diminished with maturation and the other improved with steroids (cases \#2 and 4). Amyloidosis of the external canal apparently reduces in bulk as the multiple myeloma is treated (case \#3). TB of the temporal bone has to be debrided, but also has to be treated medically (case \#1).

\section{CONCLUSION}

The anatomy of the temporal bone offers wide access to blood borne and CSF borne entry by infectious and neoplastic agents. Additional factors, perhaps a paucity of vascularization, or perhaps immune-related factors, render it particularly unable to fight infection, metastasis, or tumor neogenesis. Multiple perforations by canals, nerves, and muscles render the temporal bone structurally weak and vulnerable to fracture. Each of the pathologies addressed above required different interventions and no generalization can be made regarding the best modality of treatment for temporal bone disease.

\section{SUMMARY}

Seven cases of bilateral disease of the temporal bone are presented. These illustrate the anomalies, hematologic, and immunologic vulnerability of the temporal bone to exogenous insults. Key factors in this vulnerability are the number of fluid-filled and tissue-filled canals, as well as the robust blood supply and the thinness of the mucosal investments.

\section{REFERENCES}

[1] Hinojosa R, Redleaf MI, Green JD Jr, Blough RR. Spiral ganglion cell survival in labyrinthitis ossificans. Ann Otol Rhinol Laryngol 1995; (Suppl 166): 51-4.

[2] Redleaf MI. Cochlear implantation and temporal bone histopathology. Indian J Otol 1996; 2: 65-70.

[3] Mongkolrattnoathai K, Oram R, Redleaf M, Bova J, Englund JA. Tuberculous otitis media with central nervous system involvement. Pediatr Inf Dis J 2003; 22: 453-6.

[4] Klemens J, Baron J, Saadia-Redleaf MI. Amyloidosis of the external auditory canal. ENT J 2010; 89: 219-20. 
[5] Klemens J, Perkins J, Redleaf MI. Imaging case study of the month: sudden bilateral deafness. Ann Otol Rhinol Laryngol 2004; 113: $169-71$

[6] Redleaf MI, Pinto JM, Klemens JJ. Enlarged superior vestibular nerve canal with sensorineural hearing loss. J Laryngol Otol 2009; 123: 572-4.

[7] Brackmann DE, Doherty JP. CPA melanoma: diagnosis and management. J Otol Neurotol 2007; 28: 529-37.

[8] Goebel JA, O'Mara W, Gianoli G. Anatomic considerations in vestibular neuritis. Otol Neurotol 2001; 22: 512-8.
[9] Gianoli G, Goebel J, Mowry S, Poomipannit, P. Anatomic differences in the lateral vestibular nerve channels and their implications in vestibular neuritis. Otol Neurotol 2005; 26: 489-94.

[10] Azouz EM, Saigal G, Rodriguez MM, Podda A. Langerhans' cel histiocytosis: pathology, imaging and treatment of skeletal involvement [review]. Pediatr Rad 2005; 35: 103-15.

[11] Jacob A, Brightman R, Welling D. Bilateral cerebellopontine angle metastatic melanoma: A case report. ENT J 2007; 86: 388-90.

[12] Gerganov VM, Hore N, Herold C, et al. Bilateral malignant melanoma metastases to the internal auditory canal/cerebellopontine angle. J Neurosurg 2008; 108: 803-7.

(c) Miriam I. Saadia-Redleaf; Licensee Bentham Open.

This is an open access article licensed under the terms of the Creative Commons Attribution Non-Commercial License (http://creativecommons.org/licenses/by-nc/ $3.0 /$ ) which permits unrestricted, non-commercial use, distribution and reproduction in any medium, provided the work is properly cited. 